\title{
Incentives in cooperative networks: a contract-theoretic perspective
}

\author{
Yinshan Liu ${ }^{1,2}$, Xiaofeng Zhong ${ }^{1 *}$, Yang Yan ${ }^{1}$, Jing Wang ${ }^{1}$ and Walid Saad ${ }^{3}$
}

\begin{abstract}
Multiuser cooperative communication significantly improves the performance of wireless communication networks. One key challenge of multiuser cooperative communication is how to design a cooperative mechanism to incentivize potential relay nodes to help a source node in its data transmission. In this paper, to address this problem, a contract-based principal-agent framework is proposed in the context of a cognitive-radio-based wireless relaying networks in which the sources' wireless characteristics constitute hidden information which is not known by the relay. The problem is modeled as a monopolist's problem, in which a mobile relay node acts as the principal who designs incentive-compatible (IC) and individually rational (IR) contract items, consisting of a set of rate-price pairs. Subsequently, contract items can be broadcast by the relay to nearby mobile users that want to send data. Once these sources optimally select an item and notify the relay that they are willing to accept it, the relay then chooses one source based on the highest revenue for which to provide service. The cooperative gain, relay's revenue, and expected data rate are characterized for the optimal contract under complete information and incomplete information. Theoretical analysis and numerical results show that this pricing mechanism can lead to a win-win situation in which source nodes get good communication service and relay nodes maximize their own profit that can, in turn, be used to purchase the relay service of other nodes when needed in the future. Moreover, the proposed mechanism is shown to also exhibit other important features such as low complexity and low signaling overhead.
\end{abstract}

Keywords: Cooperative communication; Incentive mechanism; Cognitive radio; Relaying networks; Contract theory

\section{Introduction}

Multiuser cooperative communication has emerged as a promising technique for boosting the performance of wireless networks $[1,2]$. The basic premise of cooperative communications is to allow a mobile user to act as a relay node to help other neighboring mobile users via short-range communication technologies such as Wi-Fi, cellular device-to-device communications, or Bluetooth, among others. Relay has many advantages such as improving the system throughput and coverage, enhancing the link rate and reliability as well as reducing network energy consumption [3]. In addition, there is no need to upgrade existing infrastructure in order to install additional relay nodes [4]. Recent results have been shown that the capacity improves $200 \%$ and $300 \%$ for downlink and uplink respectively due to multiuser cooperation for

*Correspondence: zhongxf@tsinghua.edu.cn

1 Tsinghua National Laboratory for Information Science and Technology, Department of Electronic Engineering, Tsinghua University, Beijing, China Full list of author information is available at the end of the article cell edge users [4]. However, deploying practical cooperative communication protocols requires overcoming many technical challenges that include reducing the complexity for cooperation, managing interference, and designing suitable incentive mechanisms to facilitate relaying and cooperation [5-10].

In recent years, with the advent of highly advanced smartphones, which are capable of simultaneously supporting multi-mode radio access, including cellular network, Wi-Fi, and Bluetooth, the possibility of cooperative communication at the smartphone level has become more manageable. However, there still remains a need to design low-complexity, distributed cooperative communication mechanisms, which is both challenging and desirable. First, a serious problem of signaling overhead may increase the cost of operation in a distributed relay network. Second, mobile nodes are constrained by limited power and computational resources such as CPUs or batteries. Furthermore, while the use of relaying can improve the data rate of the source user, it can be detrimental to

\section{Springer}

(c) 2014 Liu et al.; licensee Springer. This is an Open Access article distributed under the terms of the Creative Commons Attribution License (http://creativecommons.org/licenses/by/4.0), which permits unrestricted use, distribution, and reproduction in any medium, provided the original work is properly credited. 
the relay node that must utilize its own power to transmit another node's data. Indeed, some mobile nodes have no incentive to relay the packets of other nodes due to the limitation of battery. Therefore, for such scenarios, distributed incentive mechanisms are necessary to encourage mobile relay nodes to help one another via monetary payments or credits. Here, a mobile relay node can use its earned monetary to purchase the help of other nodes when needed in the future. Thus, it is of interest to develop incentive mechanisms using which all the nodes, sources and relays, can obtain a certain benefit.

The introduction of incentive mechanisms in cooperative networks has attracted attention in the literature [5-14]. One popular approach is the use of gametheoretic techniques to introduce distributed pricing mechanisms in cooperative networks [5-8,11]. However, such approaches are often based on game-theoretic notions such as Nash bargaining that require many rounds of negotiation between the nodes which can result in a higher signaling overhead and increased complexity. Auction-theoretic algorithms (a subset of game theory) also require at least two bidder to compete a auction target $[5,6]$. Another promising approach that has recently been studied is that of contract theory [15]. Contract theory has been used to study economic contracts between service providers as well as issues related to spectrum sharing $[13,14]$ and relaying $[9,10]$. However, in the existing literature such as [9] and [10], they assume that the relay volunteer their services. The source dominates the trading process and puts a price on relay service, resulting in that the source gets the main gain of cooperation. So, the approach in [9] and [10] cannot really encourage relay node to provide help.

The main contribution of this paper is to propose a novel contract-based approach to incentivize cooperative communication in wireless networks. The key contributions include:

1. We address the problem of cooperative communication using a novel contract-based principal-agent framework to a cognitive-radiobased wireless relaying networks, in which a mobile relay node acts as the principal who designs incentive-compatible (IC) and individually rational (IR) contract items, consisting of a set of rate-price pairs.

2. We analyze the monopolistic nonlinear pricing problem under complete information and incomplete information. We first consider the complete information scenario as a benchmark and analyze the feasibility and optimality of contract under incomplete information. Then, we further characterize the solution of optimal contract in two scenarios.
3. We characterize cooperative gain (i.e., social surplus), relay's revenue, and expected data rate in the optimal contract under complete information and incomplete information. Theoretical analysis and numerical results indicate that this pricing mechanism can lead to a win-win situation in which source nodes get good communication service and the relay is encouraged since it makes most of cooperation gain from the source.

The rest of this paper is organized as follows. In Section 2, we will first present our system model and contract formulation. In Section 3, we propose the optimal contract under complete information. In Section 4, we design a contract with feasibility and optimality under incomplete information. Section 5 presents the simulation results and analysis. Finally, conclusions are drawn in Section 6.

\section{System model}

We consider a cognitive radio (CR) network that is using the underlay spectrum sharing model shown in Figure 1, which has been studied [16] without considering the two-hop relaying communication. This model consists of CR-enabled mobile stations accessing the communication network via conventional cellular base stations (BSs). In this network, some mobile stations (personal devices), acting as source nodes, seek to send data to a far BS with the help of neighboring mobile relays that have a good wireless link with its BS. The data will then be forwarded by relay to the ultimate destination of the source. However, the relays have their own data to send and may not be willing to assist the source. Therefore, it is of interest to introduce incentive mechanisms that can encourage mobile devices to relay each others data. To address this problem, we propose a novel approach based on contract theory to formulate IC and IR contract items, in which

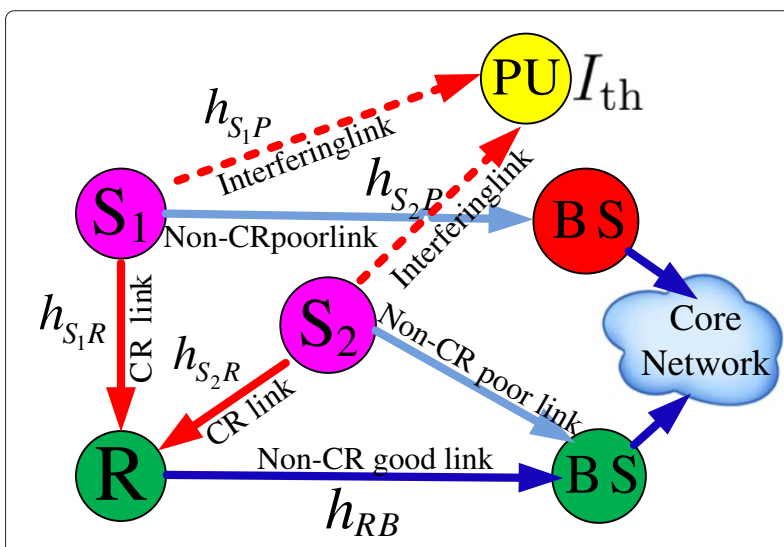

Figure 1 CR-based hybrid network architecture. 
the relay is given absolute pricing power to maximize its profit on the premise that contract will be accepted by the sources.

In our underlay sharing model, sources are allowed to adaptively select available spectrum while guaranteeing a certain quality-of-service (QoS) for the primary user (PU) as well as improving spectrum utilization effectively. The channel between the source node $S_{i}(i=1,2, \ldots, N)$ and the PU (or the relay $R$ ) is assumed to be independent slowly varying flat Rayleigh fading with variance $1 / \lambda_{S_{i} P}$ $\left(1 / \lambda_{S_{i} R}\right)$. Hence, the channel gain coefficients $\left|h_{S_{i} P}\right|^{2}$ and $\left|h_{S_{i} R}\right|^{2}$ are exponentially distributed random variable with probability density function (PDF) $f(x)=\lambda e^{-\lambda x}, \forall x \geq 0$, where $\lambda$ is $\lambda_{S_{i} P}$ or $\lambda_{S_{i} R}$, respectively [17]. The sources can share the PU's spectrum if the power of the received signal at the receiver of the PU is within a predetermined threshold $I_{t h}$ as follows: $P_{S_{i}}\left|h_{S_{i} P}\right|^{2} \leq I_{t h}$, where $P_{S_{i}}$ is the transmit power of the source $S_{i}$. Hence, the maximum transmit power of the sources is limited to $I_{t h} /\left|h_{S_{i} P}\right|^{2}$. So, the sources have different transmission rates:

$$
\theta_{S_{i}}=W \log _{2}\left(1+\frac{I_{t h}\left|h_{S_{i} R}\right|^{2}}{\sigma^{2}\left|h_{S_{i} P}\right|^{2}}\right)
$$

where $W$ is the channel bandwidth, and $\sigma^{2}$ is the variance of the white Guassian noise at the receiver. Without loss of generality, we assume $W=1$ and $\sigma^{2}$ is identical for all sources.

\subsection{Relay node model}

According to the various values of the sources' capacity, we classify the sources into different type- $\theta$ classes. We denote the set of all source types as $\Theta=[\underline{\theta}, \theta] \subseteq \Re$. The source can obtain its own type by measuring and sensing the wireless environment, while the relay is unaware of the exact type of a particular source before it formulates contract items, consisting of a set of rate-price pairs. This 'information' or 'knowledge' is private for the source or in other words 'hidden information'. Nevertheless, we assume the relay has a prior distribution over $\Theta$ given by $P(\theta)$ with a continuous density $p(\theta)>0$. Although the source informs the relay of this private information, the relay has to also consider the cost of receiving and forwarding data, which includes: cost of energy used to receive and forward data, cost of time when receiving and forwarding data, current battery level, as well as other factors.

Here, we note that the transmission rate of the CR-based link between source and relay is limited due to its transmit power constraint. If the transmission rate is too low, the relay is unwilling to serve the source for a long time since the gains from cooperation may be small. In contrast, if it is too high, the relay is unwilling to serve the source for a long time because this will reduce its profit (the cost of forwarding data via the relay will increase sharply, e.g., its transmit power must be increased exponentially to increase its data rate by two times under the constant condition of wireless environment). Thus, we define a transmission time ratio, denoted by $q \in Q=[0,1]$, as the ratio between the transmission time given by the relay and a cooperative slot. The source and the relay negotiate a contract on a pair $(q, \pi)$, with time ratio $q$ and a tariff (service price) $\pi \in \Re$. The contract is a pair of function $(q, \pi)$ : $\Theta \rightarrow Q \times \Re$ that can be viewed as a rule relating the choice of $q$ and $\pi$ about sources' type $\theta$. Here, we consider two main cost factors: $C(q)$, the cost of time for receiving data, and $G(q \theta)$, the cost of forwarding data with an average transmission rate $q \theta$ bps in a slot. It is easy to see that $C(\cdot)$ and $G(\cdot)$ are non-negative, monotone increasing, and twice differentiable. We further assume that the marginal costs $C^{\prime}(\cdot)$ and $G^{\prime}(\cdot)$ are non-decreasing, that is, $C(\cdot)$ and $G(\cdot)$ grow more rapidly at high quantities than they do at small quantities.

Therefore, the revenue $R(\theta, q)$ of a relay that is forwarding data with a rate $q \theta$ bps in a slot is given by the difference between the service price and the cost of transmission:

$$
R(\theta, q)=\pi(\theta)-C(q)-G(q \theta) .
$$

The objective of relay is thus to maximize its revenue in Equation 2.

\subsection{Source node model}

In our model, the source seeks to optimize its data rate. This rate is related not only to the capacity of the source $\theta$, in a slot, but also to the transmission time ratio $q$ allocated by a relay. Thus, we define the valuation of a type- $\theta$ source for the average transmission rate $q \theta$ bps, denoted by $V(\theta, q)$, as the utility of a source when using a relay's help. We assume $V(\theta, q)$ can simply be given by $f(q \theta)$, where $f(\cdot)$ should be monotonically increasing and concave function. Naturally, the higher the rate that is available to source, the more beneficial it is for the source. However, $f^{\prime}(\cdot)$, the marginal utility (MU) of the average transmission rate reflects the additional satisfaction that a source gains from consuming one more unit of average data rate. MU is decreasing with the increase of average transmission rate according to the law of diminishing marginal utility. Here, we can assume that $f(\cdot)$ is given by an $\alpha$-fair utility function [18]:

$$
f(q \theta)= \begin{cases}\omega(1-\alpha)^{-1}(q \theta)^{1-\alpha}, & 0 \leq \alpha<1 \\ \omega \log (q \theta), & \alpha=1 .\end{cases}
$$

where $\omega$ is the utility level of the source, which represents the source's need for the different applications [12].

It is easy to see that $V_{q}(\theta, q)=\theta f^{\prime}>0$ and $V_{\theta}(\theta, q)=$ $q f^{\prime}>0$ for all $q$, that is, every source prefers higher valuation for a given transmission time ratio, a higher type 
source has greater valuation than a lower one. We can further see that $V_{q q}(\theta, q)=\theta^{2} f^{\prime \prime}<0$, that is, $V(\theta, q)$ grows more slowly with a larger transmission time ratio. Thus, for a contract pair $(q(\theta), \pi(\theta))$, we can now define a utility function for the source as the difference between the valuation and the service price (i.e., money transfer) for the cooperative transmission:

$$
U(\theta, q)=V(\theta, q)-\pi(\theta) .
$$

We assume that each source is selfish and rational, that is seeking to always choose $q$ from a contract to maximize his utility. Thus, the optimal strategy for a type- $\theta$ source can be written as:

$$
\tilde{q}(\theta)=\arg \max _{q \in Q} V(\theta, q)-\pi(\theta) .
$$

Note that $q(\theta)=0$ means that the optimal strategy for the source is not to buy any resources from the relays.

\subsection{Formulation of contracts under incomplete information}

Contract theory studies how economic decision-maker construct contractual arrangements, generally in the presence of hidden information [15]. In our model, the sources' types constitute hidden information that is known only to the sources themselves. The relay does not know the type of each source, $\theta$, and, thus, it needs to design contract items to attract the source to take part in cooperative communications. We assume that the relay has a prior distribution $P(\theta)$ over $\Theta$. According to the revelation principle [15], for any feasible contract of incomplete information, there exists a payoff-equivalent revelation mechanism that enables the sources to truthfully report their types. Because of this, it is enough to design a optimal contract that consists of contract items $(q(\theta), \pi(\theta))$ for each source.

In this respect, we consider the optimal contract design for two information scenarios.

- Complete information. This is a benchmark case, where the relay knows each source's type. we will compute the maximum cooperative gain, which serves as an upper bound of the cooperative gain in the incomplete scenarios.

- Incomplete information. The relay does not know each source's type but has the knowledge of distribution of each type. The relay needs to design a contract to maximize its expected revenue.

Once the relay has determined the contract base on its own surplus data rate and cost of forwarding data, the interactions between the relay and sources will follow four steps.
1) The relay broadcast the contract items $(q(\theta), \pi(\theta))$ to $N$ weak sources belonging to a group, all of whom seek to benefit from cooperative with it.

2) After receiving the contract items, each weak source chooses one contract item to maximize its utility and inform the relay its choice.

3) After receiving all sources confirmations, the relay informs one selected source whose choice can make the relay to maximize its revenue.

4) The cooperative communications between the relay and the selected source begin.

\section{Optimal contract design under complete information: the benchmark scenario}

In the complete information scenario, the relay knows precisely the type of each source. We will use the maximum relay's revenue in this case to evaluate the contract performance under incomplete information.

As the relay knows source's type, it can make sure that source accepts the contract item designed for its type. In addition, due to the selfish and rational nature of the source, a source will never choose a contract that can reduce its utility beyond its reservation utility $V(\theta, 0)$. Without loss of generality, we normalize $V(\theta, 0)$ to zero. Thus, the relay needs to ensure that the source has nonnegative utility so that they are willing to accept the contract. This property allows to enforce IR on the sources. Formally, we can write the IR constraint as:

$$
V(\theta, q(\theta))-\pi(\theta) \geq 0, \quad \forall \theta \in \Theta
$$

We say a contract is optimal if it yields the maximum revenue for the relay under current information scenario. In the complete information scenario, an optimal contract can be stated as choosing a pair $(q(\theta), \pi(\theta))$ to maximize the relay's revenue for a source type of $\theta$ as follows

$$
\max _{q(\cdot), \pi(\cdot)} \pi(\theta)-C(q(\theta))-G(q(\theta) \theta)
$$

subject to the IR constraints in Equation 6.

Since relay's revenue in Equation 7 is increasing in $\pi(\theta)$, the relay can increase its revenue by increasing $\pi(\theta)$ until $V(\theta, q(\theta))-\pi(\theta)=0$ and gives source zero utility. Therefore, setting Equation 6 to equality, substituting it into Equation 7 and simplifying the relay's revenue maximization problem in Equation 7 as

$$
\max _{q(\cdot)} V(\theta, q(\theta))-C(q(\theta))-G(q(\theta) \theta) .
$$

The social surplus, denoted by $S(\theta, q)$, generated by a relay's selling of service with transmission time ratio $q$ to 
a type- $\theta$ source is defined as the aggregate utilities of both source and relay:

$$
S(\theta, q) \triangleq R(\theta, q)+U(\theta, q)=V(\theta, q)-C(q)-G(q \theta),
$$

which reflects the gain from the cooperation between source and relay. Before turning to the analysis of the solution, we define the first best solution denoted by $q^{f b}(\theta)$ as the solution of Equation 8, which would be the optimal decision (i.e., the social optimal transmission time ratio for type- $\theta$ ) if the relay found the source's type. It is easy to see that $S_{q q}(\theta, q)=V_{q q}(\theta, q)-C^{\prime \prime}(q)-\theta^{2} G^{\prime \prime}(q \theta)<$ 0 , and $q^{f b}(\theta)$ can be obtain by solving $S_{q}(q, \theta)=$ $\theta\left[f^{\prime}(q \theta)-G^{\prime}(q \theta)\right]-C^{\prime}(q)=0$ using the implicit function theorem. Thus, the maximum social surplus for each type- $\theta$ can be written as $S^{f b}(\theta)=S\left(\theta, q^{f b}(\theta)\right)$. Here, unlike traditional contract models [15] and [13] in which $q^{f b}(\theta)$ and $S^{f b}(\theta)$ are both monotone increasing with respect to $\theta$, the change trend of $q^{f b}(\theta)$ is such that it firstly increases and then decreases with the increasing of $\theta$ as shown in Figure 2. Since $q_{\theta}^{f b}(\theta)=\frac{f^{\prime}(q \theta)-G^{\prime}(q \theta)+q \theta\left[f^{\prime \prime}(q \theta)-G^{\prime \prime}(q \theta)\right]}{C^{\prime}(q)-\theta^{2}\left(f^{\prime \prime}(q \theta)-G^{\prime \prime}(q \theta)\right)}$, there exists a stationary point $q \theta=c_{0}$.

In practice, the social optimal decision may not be adopted by both the source and the relay, whose objectives are always to maximize their own utilities, without considering the social surplus. Therefore, there is a need to capture this selfish nature of the source and relay.

\section{Optimal contract design under incomplete information}

In this section, we will address the monopolist's problem and analyze the feasibility and optimality of the solution. Furthermore, the best price assignment and the best transmission time ratio assignment will be derived.
We formulate the problem as a monopolist contract design in which the principal (relay) proposes a contract pair $(q(\theta), \pi(\theta))$ to an agent (source) and, then, the source chooses a unique transmission time ratio $\tilde{q}(\theta)$ and performs a money transfer $\tilde{\pi}(\theta)$ to the relay. In general, $\pi(q(\theta))$ is simply written as $\pi(\theta)$ since $q(\theta)$ is a single-value function. A implementable contract is a set of $(q(\theta), \pi(\theta))$ such that for every type $\theta \in \Theta$, a type- $\theta$ source prefers the relaying service with transmission time ratio $q(\theta)$ at the price $\pi(\theta)$ while it does not choose any relaying service with other transmission time ratio at all. Formally, a decision function $q(\theta)$ is implementable by a money transfer $\pi(\theta)$ if the IC constraints are satisfied:

$$
V(\theta, q(\theta))-\pi(\theta) \geq V(\theta, q(\tilde{\theta}))-\pi(\tilde{\theta}), \quad \forall \theta, \tilde{\theta} \in \Theta
$$

Equivalently, the contract $(q(\theta), \pi(\theta))$ is implementable. An implementable contract that satisfies the IR constraint in Equation 6 is called feasible. In other words, a feasible contract must satisfy the IC and IR constraints.

Using the revelation principle, the monopolist's problem under incomplete information can be stated as choosing a pair $(q(\theta), \pi(\theta))$ that solves:

$$
\max _{q(\cdot), \pi(\cdot)} \int_{\Theta}[\pi(\theta)-C(q(\theta))-G(q(\theta) \theta)] p(\theta) d \theta
$$

subject to the IC and IR constraints in Equations 10 and 6.

The term $G(q \theta)$ in the cost of transmission leads to a non-standard form of the monopolist's problem. Therefore, it must be transformed into a standard form by the method of variable substitution. Making the substitution

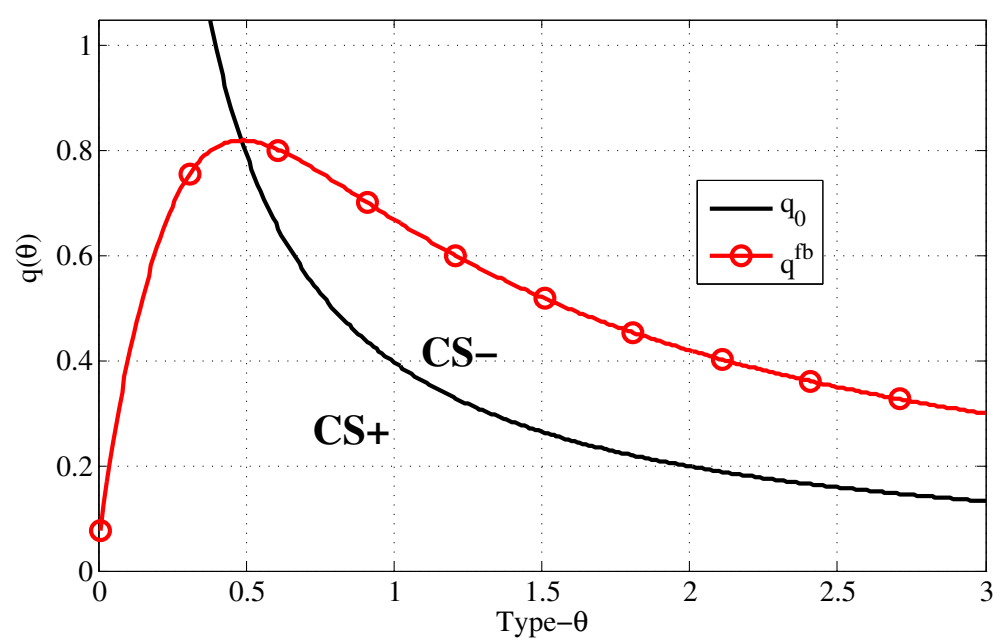

Figure 2 The first best solution $q^{f b}$ and the regions CS+ and CS-. 
$\hat{V}(\theta, q)=V(\theta, q)-G(q \theta), \hat{\pi}(\theta)=\pi(\theta)-G(q \theta)$ reduces the monopolist's problem to:

$$
\max _{q(\cdot), \hat{\pi}(\cdot)} \int_{\Theta}[\hat{\pi}(\theta)-C(q(\theta))] p(\theta) d \theta
$$

s.t.

$(\mathrm{IC})$

$\hat{V}(\theta, q(\theta))-\hat{\pi}(\theta) \geq \hat{V}(\theta, q(\tilde{\theta}))-\hat{\pi}(\tilde{\theta}), \quad \forall \theta, \tilde{\theta} \in \Theta$

$$
\hat{V}(\theta, q(\theta))-\hat{\pi}(\theta) \geq 0, \quad \forall \theta \in \Theta .
$$

\subsection{Feasibility of contract}

Furthermore, as shown in $[19,20]$, an agent's (source's) utility function $\hat{V}(\theta, q(\theta))$ in the standard monopolist's problem does not satisfy the Spence-Mirrlees conditions (SMCs) or single-crossing property (SCP), which implies that $\hat{V}_{q \theta}$ does not change sign for any value of $q$ and $\theta$. So, the local incentive compatibility constraints have to taken into account. These constraints are equivalent to the monotonicity of the decision variable with respect to the parameter $\theta$. In addition, following [20], we also relax the SMC so as to provide a simple characterization of implementability.

Definition 1. The relaxing single-crossing or SpenceMirrlees condition is the constant sign of the cross partial derivative with respect to decision and type:

(CS+) $\forall(q, \theta)$ in $Q \times \Theta: \hat{V}_{q \theta}>0$,

(CS-) $\quad \forall(q, \theta)$ in $Q \times \Theta: \hat{V}_{q \theta}<0$.

This implies that the existence of a curve $q_{0}(\theta)$ dividing the $(\theta, q)$ plane into two single-crossing regions, with $V_{q \theta}>0$ below $q_{0}$ and $V_{q \theta}<0$ above. For notational convenience, we use $\mathrm{CS}+$ and $\mathrm{CS}$ - respectively to represent these regions, as we can see in Figure 2. Under CS+ $(\mathrm{CS}-)$, higher types are associated with higher (lower) marginal valuational of the decision.

Using the implicit function theorem, we can define a unique function $q_{0}(\theta)$, such that $\hat{V}_{q \theta}=0$, i.e., $\hat{V}_{q \theta}=$ $f^{\prime}(q \theta)-G^{\prime}(q \theta)+q \theta\left[f^{\prime \prime}(q \theta)-G^{\prime \prime}(q \theta)\right] \triangleq \psi(q \theta)=0$, so $q_{0}(\theta)=\psi^{-1}(0) / \theta$, it is easy see that $q_{0}(\theta)$ is a unique decreasing function.

Remark 1. The equation $\hat{V}_{q \theta}=0$ allows to implicitly define a function $q_{0}(\theta)$ such that $\hat{V}_{q \theta}>0$ when $q<q_{0}(\theta)$ $(C S+)$ and $\hat{V}_{q \theta}<0$ when $q>q_{0}(\theta)(C S-)$.
When $\hat{V}_{q \theta}$ satisfies the relaxing single-crossing condition, one can show that implementability is equivalent to the monotonicity of the quantity assignment function, with increasing under $(\mathrm{CS}+)$ or decreasing under $(\mathrm{CS}-)$.

Lemma 1. Let $(q(\theta), \hat{\pi}(\theta))$ be an IC mechanism, then the following condition must be fulfilled:

1. $\mathcal{V}^{q}(\theta):=\hat{V}(\theta, q(\theta))-\hat{\pi}(\theta)=$ $\mathcal{V}^{q}(\underline{\theta})+\int_{\underline{\theta}}^{\theta} \hat{V}_{\theta}(\tilde{\theta}, q(\tilde{\theta})) d \tilde{\theta}, \forall \theta \in \Theta$,

2. $q(\theta)$ is non-decreasing (non-increasing) under $C S+$ $(C S-)$, i.e., $q_{\theta}(\theta) \hat{V}_{q \theta} \geq 0$.

Proof. The first part follows the envelope theorem, whose proofs can be found in [20]. By the first part in lemma, for each implementable $q(\theta)$, there exits a unique $\hat{\pi}(\theta)$ that implements it:

$$
\hat{\pi}(\theta)=\hat{V}(\theta, q(\theta))-\mathcal{V}^{q}(\theta), \quad \forall \theta \in \Theta .
$$

The global IC' in Equation 12 requires that $\mathcal{V}^{q}(\theta)-$ $\hat{V}(\theta, q(\tilde{\theta}))+\hat{\pi}(\tilde{\theta}) \geq 0$. Using Equation 13, we get $\Phi^{q}(\theta, \tilde{\theta}) \triangleq \mathcal{V}^{q}(\theta)-\hat{V}(\theta, q(\tilde{\theta}))+\hat{\pi}(\tilde{\theta})=\mathcal{V}^{q}(\theta)-\mathcal{V}^{q}(\tilde{\theta})+$ $\hat{V}(\tilde{\theta}, q(\tilde{\theta}))-\hat{V}(\theta, q(\tilde{\theta}))=\int_{\tilde{\theta}}^{\theta}\left[\int_{q(\tilde{\theta})}^{q(\hat{\theta})} \hat{V}_{q \theta}(\hat{\theta}, \hat{q}) d \hat{q}\right] d \hat{\theta} \geq 0$ (where the third equality is a consequence of the fundamental theorem of calculus), i.e., $q_{\theta}(\theta) \hat{V}_{q \theta} \geq 0$.

For the IR' constraint, if $\hat{V}_{\theta}=q\left(f^{\prime}(q \theta)-G^{\prime}(q \theta)\right)$ changes its sign, $\mathcal{V}^{q}$ in the Lemma 1 has a minimum in the interior of $\Theta$ (or at $\underline{\theta}$ ) depending on $q$ [20]. However, in order to simplify matters, we assume that it is at $\underline{\theta}$. Thus, the IR'constraint needs to be checked only at $\underline{\theta}$ and, since transfer is costly to the principal (relay), IR' must bind at $\underline{\theta}$, i.e., the IR' constraint can be replaced by $\mathcal{V}^{q}(\underline{\theta})=0$. Therefore, the condition in Lemma 1 and $\mathcal{V}^{q}(\underline{\theta})=0$ provides stricter and tighter necessary conditions for the feasible contract.

\subsection{Optimality of contract}

Among the possible feasible contracts, our objective is to find an optimal contract. We first derive the best prices for a fixed feasible transmission time ratio assignment; then, we derive the best transmission time ratio assignment for the optimal contract.

Given an implementable mechanism $(q(\theta), \hat{\pi}(\theta))$, there is a non-linear transfer $\hat{\pi}(\theta)$ that implements $q(\theta)$. And

Theorem 1. Let $(q(\theta), \hat{\pi}(\theta))$ be a feasible contract with the fixed decision variable assignment $q(\theta)$. Then, the unique best price assignment is given by:

$$
\hat{\pi}(\theta)=\hat{V}(\theta, q(\theta))-\int_{\underline{\theta}}^{\theta} \hat{V}_{\theta}(\tilde{\theta}, q(\tilde{\theta}) d \tilde{\theta} .
$$


Proof. By Lemma 1, for each implementable $q(\theta)$, there exits a unique

$$
\hat{\pi}(\theta)=\hat{V}(\theta, q(\theta))-\mathcal{V}^{q}(\underline{\theta})-\int_{\underline{\theta}}^{\theta} \hat{V}_{\theta}(\tilde{\theta}, q(\tilde{\theta})) d \tilde{\theta} .
$$

Furthermore, according to IR' condition, we have $\mathcal{V}^{q}(\underline{\theta})=0$. Plugging it into the above equation completes the proof.

Thus, as the unique best price assignment for the feasible contract $(q(\theta), \hat{\pi}(\theta))$ has been given, we can make a transformation back into terms of $\pi(\theta)$ by making the substitution $\hat{V}(\theta, q)=V(\theta, q)-G(q \theta), \hat{\pi}(\theta)=\pi(\theta)-G(q \theta)$. As a result, we obtain the following corollary.

Corollary 1. The unique best price assignment for the feasible contract $(q(\theta), \pi(\theta))$ is given by:

$$
\pi(\theta)=V(\theta, q(\theta))-\int_{\underline{\theta}}^{\theta}\left[V _ { \theta } \left(\tilde{\theta}, q(\tilde{\theta})-G_{\theta}(\tilde{\theta}, q(\tilde{\theta})] d \tilde{\theta} .\right.\right.
$$

Theorem 1 suggests that, for any fixed feasible decision variable assignment $q(\theta)$, the best price assignment given by Equation 14 is unique. Therefore, for the monopolist's problem, we plug Equation 14 into the objective function (Equation 12) to find:

$$
\begin{aligned}
& \int_{\Theta}[\hat{\pi}(\theta)-C(q(\theta))] p(\theta) d \theta \\
= & \int_{\Theta}\left[\hat{V}(\theta, q(\theta))-C(q(\theta))-\int_{\underline{\theta}}^{\theta} \hat{V}_{\theta}(\tilde{\theta}, q(\tilde{\theta}) d \tilde{\theta}] p(\theta) d \theta .\right.
\end{aligned}
$$

Integrating by parts, we have $\int_{\Theta} h(\theta, q(\theta)) p(\theta) d \theta$, where $h(\theta, q(\theta))=\hat{V}(\theta, q(\theta))-C(q(\theta))-\frac{1-P(\theta)}{p(\theta)} \hat{V}_{\theta}(\theta, q(\theta))$ is the virtual surplus. Thus, we consider the IC' and IR' constraints and denote $q^{s b}(\theta)$ as the solution which maximizes the following relaxed problem:

$$
\max _{q(\cdot)} \int_{\Theta} h(\theta, q(\theta)) p(\theta) d \theta
$$

which can be reduced to a pointwise maximization of the $h(\theta, q(\theta))$. Obviously, $q^{s b}(\theta)$ can be found at the boundary points $(\underline{\theta}$ and $\bar{\theta})$ or at the critical point, i.e., according to Fermat's theorem for stationary points, $q^{s b}(\theta)$ satisfying $h_{q}\left(\theta, q^{s b}(\theta)\right)=0$ and $h_{q q}\left(\theta, q^{s b}(\theta)\right) \leq 0$, for all $\theta \in \Theta$. It is easy to see that the virtual surplus $h(\theta, q(\theta))$ is the social surplus $(\hat{V}-C)$ discounted by the agent's information rent $-\frac{1-P}{p} \hat{V}_{\theta}$.

Proposition 1. The optimal decision $q^{f b}$ satisfies the second term condition in the Lemma 1, i.e., $q_{\theta}^{f b}(\theta) \hat{V}_{q \theta} \geq 0$.
Proof. From the previous calculations, we know that $\hat{V}_{q \theta}=f^{\prime}(q \theta)-G^{\prime}(q \theta)+q \theta\left[f^{\prime \prime}(q \theta)-G^{\prime \prime}(q \theta)\right]$, and $q_{\theta}^{f b}(\theta)=\frac{\hat{V}_{q \theta}}{C^{\prime}(q)-\theta^{2}\left(f^{\prime \prime}(q \theta)-G^{\prime \prime}(q \theta)\right)}$, whose denominator is non-negative. So, $q_{\theta}^{f b} \hat{V}_{q \theta} \geq 0$.

This proposition suggest that the solution $q^{f b}$ of the maximum social surplus is implementable. However, the $q^{s b}$ may be unimplementable since the agent's information rent $-\frac{1-P}{p} \hat{V}_{\theta}$ in the virtual surplus depends on the distribution of source types. If the $q^{s b}(\theta)$ is implementable, then it is the solution of the problem (Equation 12) subject to IC' and IR' constrains. Therefore, under the SMC, $q^{s b}(\theta)$ is the solution of the problem (Equation 12) if and only if it is monotonic. Otherwise, the monotonicity condition $q_{\theta}(\theta) \hat{V}_{q \theta} \geq 0$ in Lemma 1 is binding. When $q_{\theta}(\theta) \hat{V}_{q \theta} \geq 0$ is binding, one has to respectively perform the ironing principle [20] on $q^{s b}(\theta)$ under the region CS+ and CS- to make the infeasible region to be feasible (for a complete analysis of the standard case, see [15]). Using this principle, we design a dynamic algorithm too. The detailed algorithm is shown in Algorithm 1, where an infeasible region $\left[\theta_{1}, \theta_{2}\right]$ is defined as a subset of $\Theta$, such that $\forall \theta \in$ $\left[\theta_{1}, \theta_{2}\right]$, the monotonicity condition $q_{\theta}(\theta) \hat{V}_{q \theta} \geq 0$ is violating. By ironing algorithm, we get a feasible solution of the problem (Equation 12).

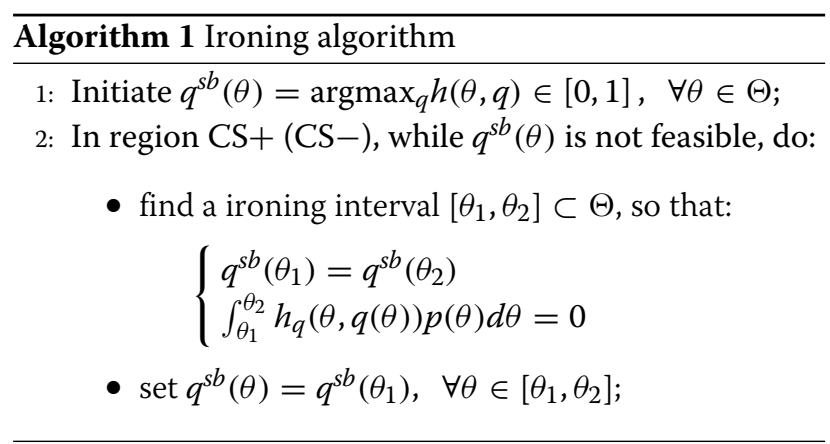

\subsection{Multiple-source selection and revenue}

In the last section, we applied the contract-based principal-agent framework to a cognitive-radio-based wireless relaying networks, in which a mobile relay acts as the principal who designs IC and IR contract items $(q(\theta), \pi(\theta))$, which are then broadcast to $N$ weak sources. After receiving all sources confirmations, the relay needs to choose one source who can make the relay to maximize its revenue. Obviously, the higher the source type, the more revenue got by relay. From Lemma 1, it can be seen that the transmission time ratio assignments $q^{s b}$ in the optimal contracts are non-increasing under the region CS-. This means that the higher source type- $\theta$ (exceeding 
a threshold $\theta_{1}$ or $\theta_{2}$, when $\theta$ blows the threshold, $q^{s b}=0$ as shown in Figure 3, denotes that relay receives zero revenue) will obtain the less transmission time ratio assignments. Moreover, the same cost of forwarding data, the relay prefers to service for high source type with more less transmission time assignments. Thus, the relay will naturally choose a high type source who owns the maximum transmission rate among all weak sources.

We now calculate the revenue generated by the relay, keeping in mind that the relay's revenue can change with the number of sources $N$ and probability distribution of $\theta_{i}(i=1,2, \ldots, N)$. The revenue obtained from optimal contract is the key parameter that stimulates cooperative in the relaying network. In our cognitive-radio-based underlay sharing model, all channels are assumed to be independent and identically slowly varying flat Rayleigh fading channel. According to [21] and the transformation of random variables, the PDF of $\theta_{i}$ is then given as

$$
p_{\theta_{i}}(x)=\frac{\frac{\lambda_{i P} I_{t h}}{\lambda_{i R} \sigma^{2}} 2^{x} \log 2}{\left(2^{x}-1+\frac{\lambda_{i P} I_{t h}}{\lambda_{i R} \sigma^{2}}\right)^{2}},
$$

and the cumulative density function (CDF) of $\theta_{i}$ is given by

$$
P_{\theta_{i}}(x)=\frac{2^{x}-1}{2^{x}-1+\frac{\lambda_{i P} I_{t h}}{\lambda_{i R} \sigma^{2}}} .
$$

Let us introduce some notations. Given the number of sources $N$ and the PDF of type- $\theta_{i}$, the revenue accrued by the relay from the optimal contract $(q(\theta), \pi(\theta))$ is denoted by $E(R)$, the social surplus accrued by the source and relay is denoted by $E(S)$, and expected average transmission rate obtained by all sources is denoted by $E(q \theta)$.
Theorem 2. The expected revenue $E(R)$, excepted social surplus $E(S)$, and expected average rate $E(q \theta)$ from the optimal contract $(q(\theta), \pi(\theta))$ with $N$ sources under both complete information and incomplete information are given by

$$
\begin{aligned}
& E(R)=N E_{\theta}\left[R(\theta, q(\theta)) P_{\theta}^{N-1}(\theta)\right], \\
& E(S)=N E_{\theta}\left[S(\theta, q(\theta)) P_{\theta}^{N-1}(\theta)\right], \\
& E(q \theta)=N E_{\theta}\left[q(\theta) \theta P_{\theta}^{N-1}(\theta)\right] .
\end{aligned}
$$

Proof. Define $\Omega_{1}$ as the event that the source $S_{1}$ purchases the relay service from the competition of $\mathrm{N}$ sources. The probability that source $S_{1}$ possesses the highest transmission rate is given by

$$
\begin{aligned}
\operatorname{Pr}\left(\Omega_{1}\right) & =\operatorname{Pr}\left(\theta_{2} \leq \theta_{1}, \ldots, \theta_{N} \leq \theta_{1}\right) \\
& =\int_{\Theta}\left[P_{\theta_{2}}\left(x_{1}\right)\right]^{N-1} p_{\theta_{1}}\left(x_{1}\right) d x_{1}=E_{\theta_{1}}\left[P_{\theta_{2}}^{N-1}\left(\theta_{1}\right)\right] .
\end{aligned}
$$

The expected revenue for the seller from source $S_{1}$ alone is derived next. The conditional payment made by the highest type source $S_{1}$ can be written as

$$
\begin{aligned}
& E_{\theta_{1}}\left.R\left(\theta_{1}, q\left(\theta_{1}\right)\right) \mid \theta_{2} \leq \theta_{1}, \ldots, \theta_{N} \leq \theta_{1}\right) \operatorname{Pr}\left(\Omega_{1}\right) \\
& \quad= \int_{\Theta} R\left(\theta_{1}, q\left(\theta_{1}\right) N\left[P_{\theta_{2}}\left(x_{1}\right)\right]^{N-1} p_{\theta_{1}}\left(x_{1}\right) d x_{1} \operatorname{Pr}\left(\Omega_{1}\right)\right. \\
& \quad=N E_{\theta_{1}}\left[R\left(\theta_{1}, q\left(\theta_{1}\right) P_{\theta_{2}}^{N-1}\left(\theta_{1}\right)\right] \operatorname{Pr}\left(\Omega_{1}\right) .\right.
\end{aligned}
$$

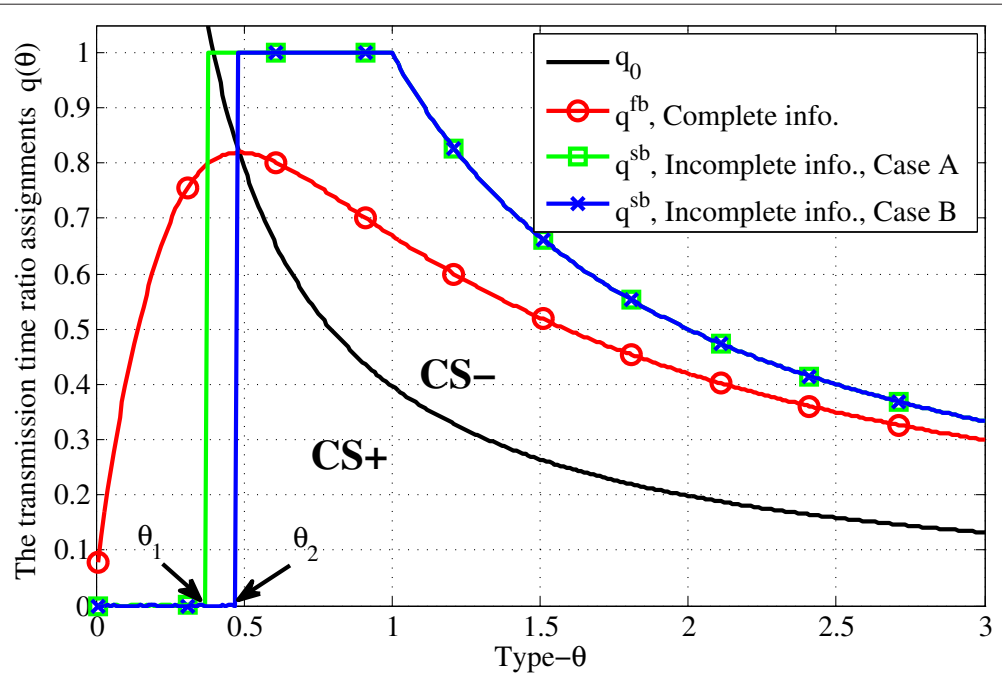

Figure 3 The transmission time ratio and price assignments in the optimal contracts. 
Therefore, using Equations 24 and 25, the expected revenue at the relay averaged over all $N$ sources is

$$
E(R)=N^{2} E_{\theta_{1}}\left[P_{\theta_{2}}^{N-1}\left(\theta_{1}\right)\right] E_{\theta_{1}}\left[R\left(\theta_{1}, q\left(\theta_{1}\right) P_{\theta_{2}}^{N-1}\left(\theta_{1}\right)\right]\right.
$$

Under the i.i.d channel assumption, using $P_{\theta_{2}}=P_{\theta_{1}}$, we obtain $E_{\theta_{1}}\left[P_{\theta_{2}}^{N-1}\left(\theta_{1}\right)\right]=1 / N$, substituting in to Equation 26 and omitting the subscript of $\theta_{i}$, we obtain the result of Equation 21.

Similarity, we can obtain the results of Equations 22 and 23.

\section{Simulation results}

Here we use numerical results to show the performance of the proposed contracts in different environments. In a common environment, two different distributions of type $\theta$ for purposes of research will be investigated on the contract effect of different distributions. In the Rayleigh fading channel environment, we will capture the cooperative gain and performance improvement with respect to the predetermined threshold $I_{t h}$ and the number of sources.

\subsection{A common environment}

In this environment, we implement the proposed contract in a continuous-type model. A source type $\theta$ is distributed with a probability distribution $p(\theta)$ on the interval $[0,3]$. In our simulations, parameters $\alpha$ and $\omega$ of utility function are set to 0.5 and 1 , respectively. The cost functions of the relay are defined as $C(q)=0.5 q^{1.2}$ and $G(q \theta)=$ $0.5(q \theta)^{2}$. We investigate the optimal contracts in two scenarios which differ from one another in the distribution of type. In case $\mathrm{A}$, all types are uniformly distributed on the $[0,3]$ with $p(\theta)=1 / 3$; in case $\mathrm{B}$, the large type has larger probability than the small type, where $p(\theta)=2 \theta / 9$.

Figures 3 and 4 present the transmission time ratio and the best price assignments in the optimal contracts, respectively. In Figure 3, the curve with circle represents the social optimal transmission time ratio assignments under complete information, i.e., the first best solution $q^{f b}$, which maximizes the social surplus. The curves with square and cross denote the optimal transmission time ratio assignments $q^{s b}$ in the optimal contracts under incomplete information for scenarios $\mathrm{A}$ and $\mathrm{B}$, respectively. It can be seen that $q^{s b}$ is non-decreasing (nonincreasing) under the region $\mathrm{CS}+(\mathrm{CS}-)$, satisfies the second term condition in Lemma 1 . Note, for example, in case $\mathrm{A}$, where $q(\theta)=1$ denotes that the transmission time ratio assignments reach the maximum, where $q(\theta)=0$, i.e., $\theta \in\left[0, \theta_{1}\right]$, denotes an aborted trading process; when $\theta \in\left(\theta_{1}, 3\right]$, the transmission time ratio assignment in the optimal contract under incomplete information is always more than the social optimal ratio assignment under complete information. This can be explained as follows, for the propose of revenue maximizing, the relay will reduce the supply to the lower type source and increase the time assignment to the higher type source. Meanwhile, it charges a much higher price from the source. Furthermore, if the probability of lower type sources becomes smaller, e.g., case B, the relay tends to reduce more supply on the low type sources and charges at higher price from the higher type sources, and the source gets a much higher data rate as shown in Figure 5. For the optimal contract under complete information, the average data rate is monotonically increasing with respect to $\theta$. However, for an optimal contract under incomplete

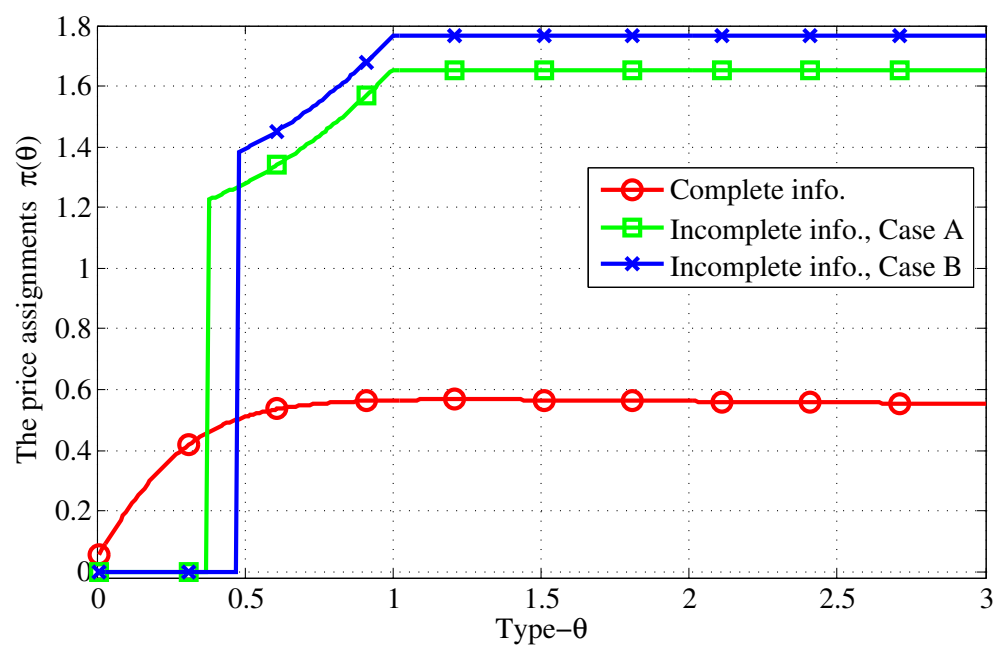

Figure 4 The price assignments in the optimal contracts. 


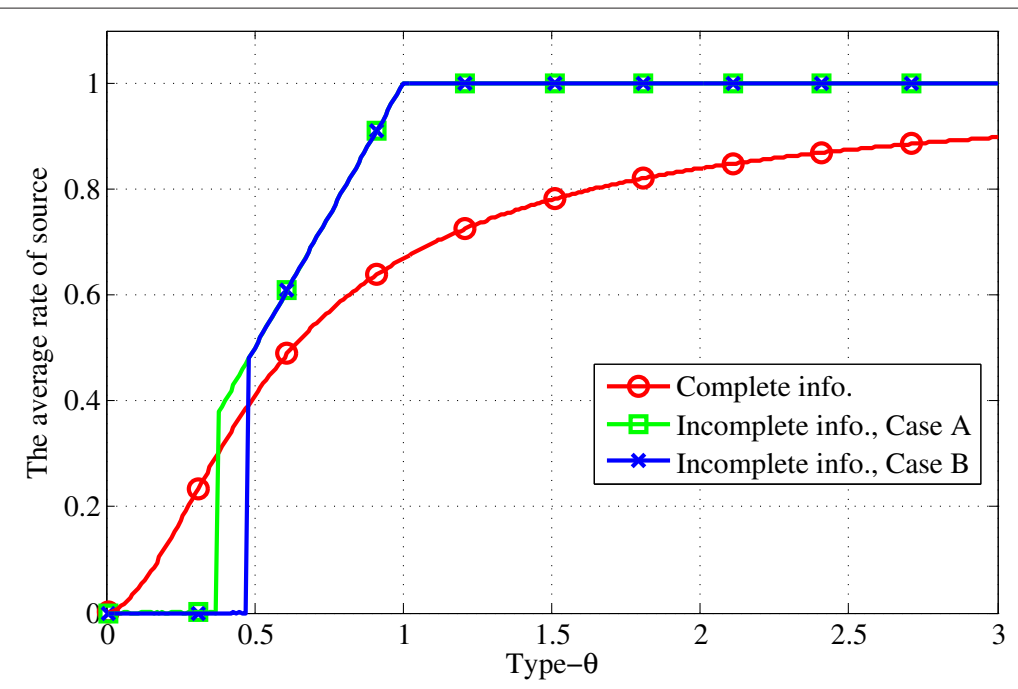

Figure 5 The average rate of source in the optimal contracts.

information in both cases $\mathrm{A}$ and $\mathrm{B}$, the average data rate is zero when instant transmission rate $\theta$ is small, while when instant transmission rate $\theta$ is big, it is bigger than that of optimal contract under complete information result from the changing of the transmission time ratio assignments.

Figure 6 shows the social surplus and the revenues of the relay in the optimal contracts. In this histogram, the 1st and 2nd bar denote the social optimal surplus and the revenue of the relay in the social optimal contract under complete information, respectively, while the 3rd and 4th bar denote the social surplus and the revenue of the relay in the optimal contracts under incomplete information, respectively. Clearly, social surplus under incomplete information is only slightly smaller than that under complete information, which reflects the upper bound of cooperation gain. In Figure 6, we can see that the relay gains more revenue from the optimal contract under incomplete information, at the expense of social surplus decreasing caused by the dropping out of low type sources. i.e., a region of $q^{s b}=0$. The selfish behavior of the relay will decrease the social surplus in a short-term, but it may lead to a higher expected revenue in a long-term. Furthermore, the social surplus in case B is more than that in case A result from the probability of higher type sources becomes larger in case $\mathrm{B}$, which reflects that the more gain of cooperation comes from the more higher type sources.

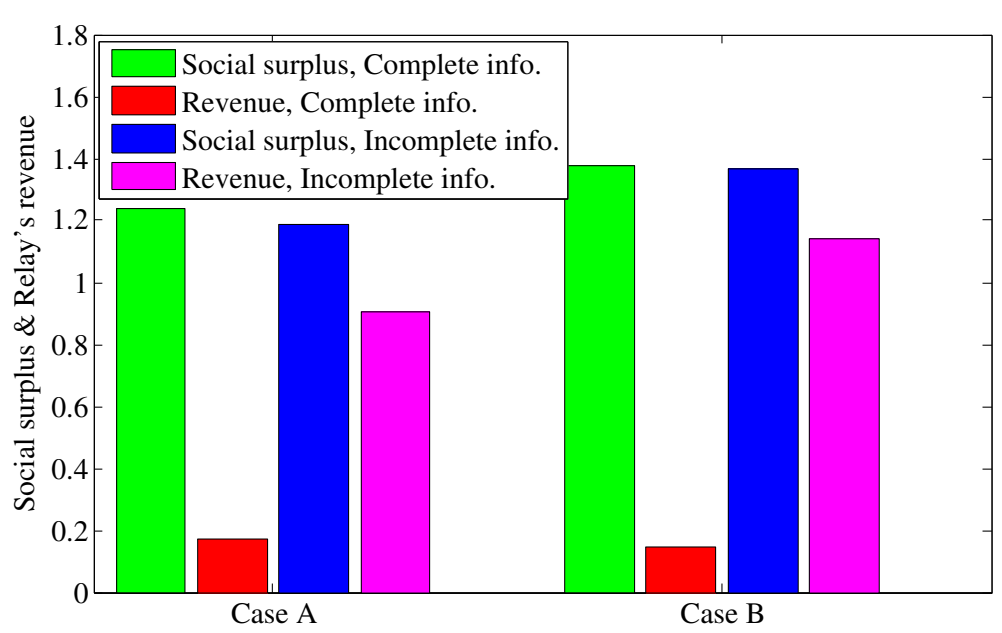

Figure 6 The social surplus and the revenues of the relay in the optimal contracts. 


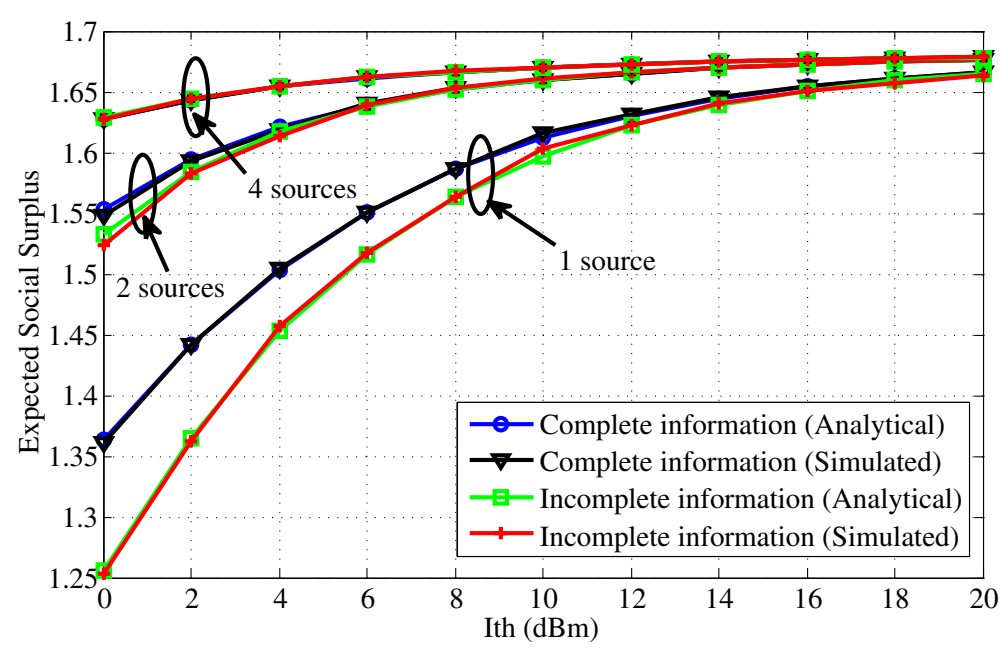

Figure 7 Expected social surplus in the optimal contracts under complete information and incomplete information.

\subsection{The Rayleigh fading channel environment}

In the Rayleigh fading channel environment, the simulation parameters are set as follows: the channel gain parameters are $\lambda_{S_{i} P}=1$ and $\lambda_{S_{i} R}=1$; the noise variance is $\sigma^{2}=0 \mathrm{dBm}$. The predetermined threshold $I_{t h}$ varies from $0 \sim 20 \mathrm{dBm}$.

Figure 7 displays the social surplus in the optimal contracts under complete information and incomplete information with respect to the predetermined threshold $I_{t h}$ for different number of sources $N$. It can be seen that the simulated social surplus curves are in agreement with derived analytical results. And the cooperative gain improves with increasing of the interference temperature of a primary receiver $I_{t h}$. This is mainly due to when $I_{t h}$ becomes larger, allowing very high transmit power levels, i.e., sources own very high type. Additionally, the cooperative gain under incomplete information is only slightly smaller than that under complete information for 1,2 , and 4 sources, respectively. Moreover, the cooperative relaying system gets higher diversity gain with a greater number of sources, when the $I_{t h}$ is low.

Figure 8 displays the seller revenue accrued by the relay. It can be seen that the simulated revenue curves are in agreement with derived analytical results under complete information and incomplete information for 1, 2, and 4 sources, respectively. Furthermore, we can see that the relay gains more revenue from the optimal contract under incomplete information. More specifically, the revenue

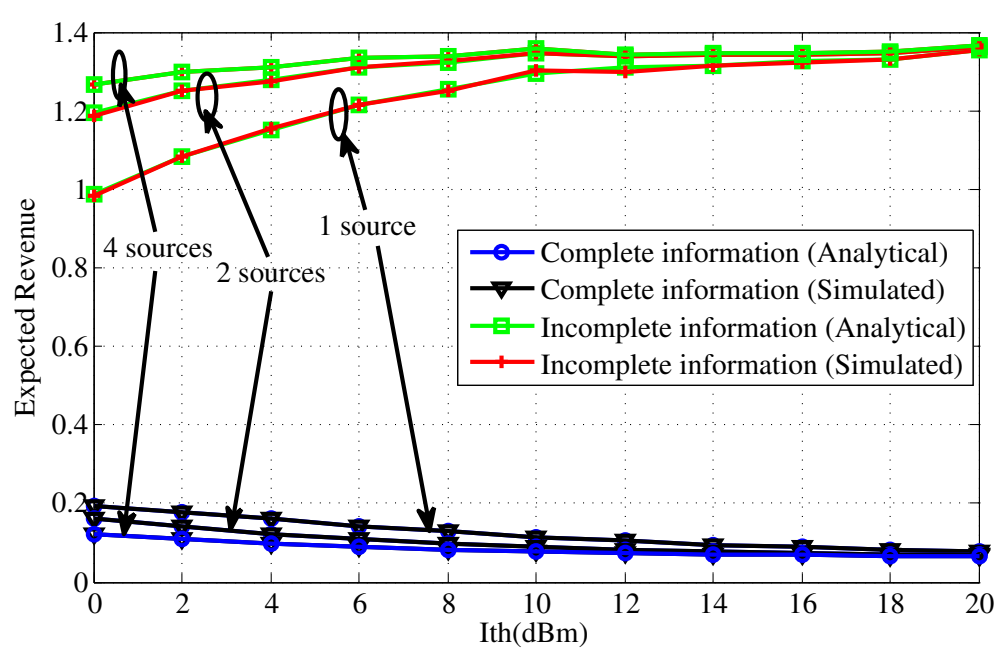

Figure 8 Expected relay's revenue in the optimal contracts under complete information and incomplete information. 


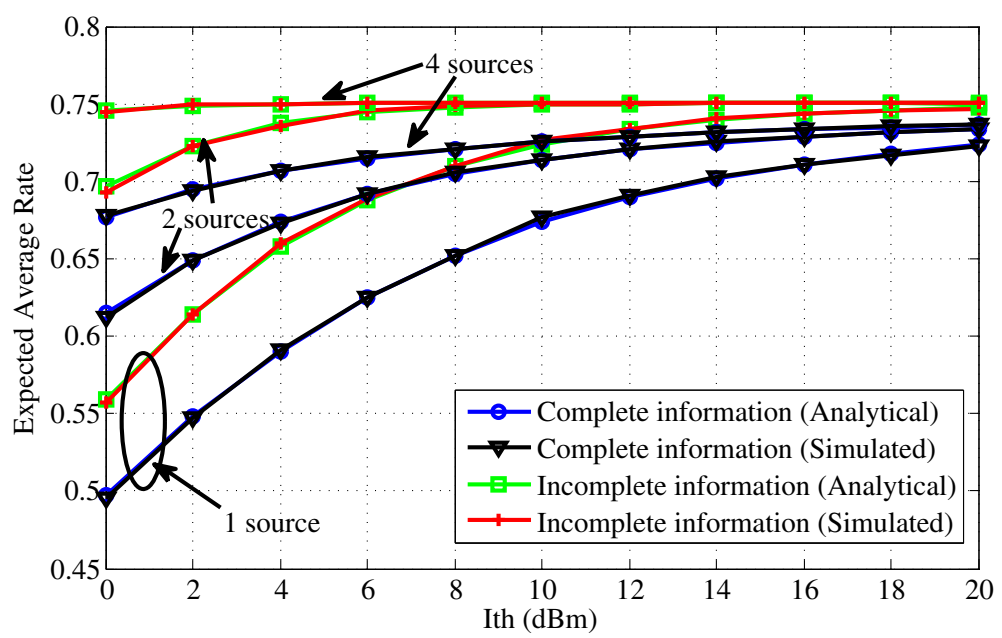

Figure 9 Expected average rate in the optimal contracts under complete information and incomplete information.

obtained by the relay is at least $77 \%$ in social surplus under incomplete information, while it is no more than $15 \%$ in social surplus under complete information.

Figure 9 shows expected average rate of all sources, It can be seen that the simulated average-rate curves are in agreement with derived analytical results under complete information and incomplete information for 1 , 2 , and 4 sources, respectively. Furthermore, we can see that the expected average rate under incomplete information outperforms that under complete information, since the higher type source gains more transmission time ratio assignments and spends with a much higher price.

\section{Conclusion}

In this paper, we have studied a pricing mechanism for multiuser cooperative communication in a cognitiveradio-based wireless network using a simple principalagent framework, in which the relay acts as a principal who designs contract items and sources act as the agent who purchases the relaying service of sources. In this model, the contract items designed by the principal consist of a set of rate-price pairs. We have studied the optimal contract design under complete information and incomplete information. We have also analyzed the feasibility and optimality of the feasible contract under incomplete information and we have derived the best variable assignment and the best price assignment. Further, we have characterize cooperative gain, relay's revenue, and expected data rate in the optimal contract under complete information and incomplete information. The proposed mechanism is simple and requires limited interaction between source and relay. Numerical simulation results have shown that this pricing mechanism can lead to a win-win situation, where the source nodes get good communication service and the relay nodes maximize their own profit. From a social surplus perspective, our results have shown that the social surplus under incomplete information is close to the maximum social surplus under complete information.

\section{Competing interests}

The authors declare that they have no competing interests.

\section{Acknowledgements}

This work is supported by the National Basic Research Program of China (2012CB316000), National Natural Science Foundation of China (61201192), National S\&T Major Project (2015ZX03002010-002), International Science and Technology Cooperation Program(2012DFG12010), Open Research Fund of National Mobile Communications Research Laboratory, Southeast University (2012D02), Cooperation Agreement Between THU and SEC, and, in part, by the U.S. National Science Foundation under grant CNS-1513697.

A preliminary version of this paper was presented in part at the 9th

International Conference on Wireless Algorithms, Systems, and Applications (WASA 2014), Harbin, China.

\section{Author details}

${ }^{1}$ Tsinghua National Laboratory for Information Science and Technology, Department of Electronic Engineering, Tsinghua University, Beijing, China.

${ }^{2}$ Dalian Airforce Communication NCO Academy, Dalian, Liaoning, China.

3Wireless@VT, Bradley Department of Electrical and Computer Engineering,

Virginia Tech, Blacksburg, VA, USA.

Received: 2 September 2014 Accepted: 24 November 2014

Published: 4 December 2014

\section{References}

1. A Sendonaris, E Erkip, B Aazhang, User cooperation diversity. Part I. System description. Commun. IEEE Trans. 51(11), 1927-1938 (2003)

2. A Sendonaris, E Erkip, B Aazhang, User cooperation diversity. Part II. Implementation aspects and performance analysis. Commun. IEEE Trans. 51(11), 1939-1948 (2003)

3. X Cheng, DZ Du, L Wang, B Xu, Relay sensor placement in wireless sensor networks. Wireless Netw. 14(3), 347-355 (2008)

4. KVanganuru, S Ferrante, G Sternberg, in MILITARY COMMUNICATIONS CONFERENCE-MILCOM. System capacity and coverage of a cellular network with D2D mobile relays (IEEE Piscataway, 2012), pp. 1-6 
5. J Huang, Z Han, M Chiang, HV Poor, Auction-based resource allocation for cooperative communications. Selected Areas Commun. IEEE J. 26(7), 1226-1237 (2008)

6. A Mukherjee, HM Kwon, General auction-theoretic strategies for distributed partner selection in cooperative wireless networks. Commun. IEEE Trans. 58(10), 2903-2915 (2010)

7. Z Han, Game theory in wireless and communication networks: theory, models, and applications. (Cambridge University Press, New York, 2012)

8. B Wang, Z Han, KR Liu, in INFOCOM 2007. 26th IEEE International Conference on Computer Communications. Distributed relay selection and power control for multiuser cooperative communication networks using buyer/seller game (IEEE Piscataway, 2007), pp. 544-552

9. Z Hasan, A Jamalipour, VK Bhargava, in Wireless Communications and Networking Conference (WCNC), 2012 IEEE. Cooperative communication and relay selection under asymmetric information (IEEE Piscataway, 2012), pp. 2373-2378

10. B Nazari, A Jamalipour, in Communications in China (ICCC), 2012 1st IEEE International Conference on. Contract design for relay-based cooperative communication with hidden channel state information (IEEE Piscataway, 2012), pp. 798-803

11. DE Charilas, AD Panagopoulos, A survey on game theory applications in wireless networks. Comput. Netw. 54(18), 3421-3430 (2010). doi:10.1016/j.comnet.2010.06.020

12. P Hande, M Chiang, R Calderbank, J Zhang, in INFOCOM, 2010 Proceedings IEEE. Pricing under constraints in access networks: Revenue maximization and congestion management (IEEE Piscataway, 2010), pp. 1-9

13. L Duan, L Gao, J Huang, in New Frontiers in Dynamic Spectrum Access Networks (DySPAN), 2011 IEEE Symposium on. Contract-based cooperative spectrum sharing (IEEE Piscataway, 2011), pp. 399-407

14. L Gao, X Wang, Y Xu, Q Zhang, Spectrum trading in cognitive radio networks: A contract-theoretic modeling approach. Selected Areas Commun. IEEE J. 29(4), 843-855 (2011)

15. P Bolton, M Dewatripont, Contract theory. (MIT press, Cambridge, $M A, 2005)$

16. Z Cai, S Ji, J He, L Wei, A Bourgeois, Distributed and asynchronous data collection in cognitive radio networks with fairness consideration. Parallel Distributed Syst. IEEE Trans. 25(8), 2020-2029 (2014)

17. T Jing, $X$ Chen, $Y$ Huo, $X$ Cheng, in INFOCOM, 2012 Proceedings IEEE. Achievable transmission capacity of cognitive mesh networks with different media access control (IEEE Piscataway, 2012), pp. 1764-1772

18. L Yang, H Kim, J Zhang, M Chiang, CW Tan, Pricing-based decentralized spectrum access control in cognitive radio networks. IEEE/ACM Trans. Netw. (TON). 21(2), 522-535 (2013)

19. I Segal, Lecture notes in contract theory. (Department of Economics, Stanford University, 2010)

20. A Araujo, $\mathrm{H}$ Moreira, Adverse selection problems without the Spence-Mirrlees condition. J. Econ. Theory. 145(3), 1113-1141 (2010)

21. Y Guo, G Kang, N Zhang, W Zhou, P Zhang, Outage performance of relay-assisted cognitive-radio system under spectrum-sharing constraints. Electron. Lett. 46(2), 182-184 (2010)

doi:10.1186/1687-1499-2014-211

Cite this article as: Liu et al:: Incentives in cooperative networks: a contract-theoretic perspective. EURASIP Journal on Wireless Communications and Networking 2014 2014:211.

\section{Submit your manuscript to a SpringerOpen ${ }^{\odot}$ journal and benefit from:}

- Convenient online submission

Rigorous peer review

- Immediate publication on acceptance

- Open access: articles freely available online

- High visibility within the field

- Retaining the copyright to your article

Submit your next manuscript at $\boldsymbol{\triangleright}$ springeropen.com 